\title{
Herlyn-Werner-Wunderlich Syndrome with Central Precocious Puberty: A Case Report
}

\author{
Jeeho Han, M.D. \\ Jae Man Lee, M.D. \\ Geon Hee Kim, M.D. \\ Su Jin Kim, M.D. \\ Department of Pediatrics, Myongji \\ Hospital, Hanyang University College \\ of Medicine \\ Corresponding author: \\ Su Jin Kim, M.D. \\ Address: Department of Pediatrics, \\ Myongji Hospital, Hanyang University \\ college of Medicine, 55 Hwasu-ro \\ 14beon-gil, Deogyang-gu, Goyang-si \\ 10475, Gyeonggi-do, Republic of Korea \\ Tel: +82-31-810-5114 \\ Fax: +82-31-962-4902 \\ E-mail:zzzing78@hanmail.net \\ Received: 29 August 2019 \\ Revised: 15 October 2019 \\ Accepted: 18 October 2019
}

Herlyn-Werner-Wunderlich (HWW) syndrome is a rare congenital anomaly of the genitourinary tract comprising uterus didelphys, obstructed hemivagina, and ipsilateral renal agenesis. Patients with HWW syndrome usually present symptoms such as dysmenorrhea, abdominal pain, pelvic mass, and purulent vaginal discharge. If not treated at an appropriate time, complications such as infertility, endometriosis, pyosalpinx, and subsequent pelvic adhesions may occur. Here, we report a case of HWW syndrome in a 7-year-old-girl who was also diagnosed as having central precocious puberty. She was brought to the pediatric department with chief complaints of lump in her breast and vaginal discharge. When she was around 2 months old, she was confirmed to have a single kidney on ultrasonography. We checked her past medical history and diagnosed her as having HWW syndrome based on the results of imaging studies, including abdominal ultrasonography and pelvic magnetic resonance imaging. She underwent treatment with gonadotropin-releasing hormone analogue for 2 years. During 24 months of follow-up, she showed no serious problems or complications. If renal anomalies are identified immediately after birth or in infancy, further screening tests should be conducted prior to menstruation for determining congenital abnormalities of the reproductive tract and vice versa.

Key words: HWW syndrome, Central precocious puberty, Renal agenesis, Hemivagina, Uterus didelphys

\section{Introduction}

Herlyn-Werner-Wunderlich (HWW) syndrome is a rare congenital anomaly of the genitourinary tract characterized by the classic triad that includes uterus didelphys, obstructed hemivagina, and ipsilateral renal agenesis. ${ }^{1)}$

Patients with HWW syndrome usually present symptoms such as dysmenorrhea, abdominal pain, pelvic mass, and purulent vaginal discharge ${ }^{2)}$. Symptoms usually occur at the age of 12-13 years, immediately after the onset of menarche, and most patients are diagnosed from 2 months to 1 year after menarche $e^{3)}$. If not treated at an appropriate time, complications such as infertility, endometriosis, pyosalpinx, and subsequent pelvic adhesions can develop ${ }^{4,5)}$. Therefore, diagnosis and treatment of HWW syndrome at an early stage are important.

We report the case of a 7-year-old girl with central precocious puberty who was early diagnosed as having HWW syndrome through further diagnostic 
assessments.

\section{Case report}

A 2 months old female baby was brought to the pediatric emergency department of our hospital, with cough as the single chief complaint. She had no fever, and was in good overall condition. In her past history, she had been healthy at birth, with a gestational age of 38 weeks and with a birth weight of 2,760 g. Also abnormal findings were not observed during prenatal examinations. Laboratory investigation conducted by our hospital confirmed increased levels of transaminases (aspartate aminotransferase, 395 IU/L and alanine aminotransferase, 198 IU/L), and abdominal ultrasonography was performed for further evaluation, which revealed left kidney agenesis and right kidney compensatory hypertrophy. No remarkable findings from the liver and spleen were observed. She was diagnosed with bronchitis, reactive hepatitis and left kidney agenesis, and the progress was improved after 5 days of conservative management. The need for regular outpatient follow-up was discussed with her parents, but they did not comply. Since she had not visited the hospital for a long time, further examinations including Dimercaptosuccinic acid (DMSA) scan and Voiding cystourethrography (VCUG) were not conducted.

7 years after the first visit, she revisited the pediatric de- partment with a chief complaint of lump in her breast and vaginal discharge. On physical examination, her height was $130.4 \mathrm{~cm}$ (90th percentile) and her weight was $34.3 \mathrm{~kg}$ (7590th percentile). Her mother's and father's heights were 163 and $183 \mathrm{~cm}$, respectively. Her breast development was Tanner stage II on sexual maturity rating, and her bone age showed 10 years old using the standards of Greulich-Pyle method, which is advanced compared to chronological age. A gonadotropin-releasing hormone $(\mathrm{GnRH})$ (gonadorelin $100 \mu$ g, Relefact LH-RH, LG chem, Seoul, Korea ) stimulation test was done. Laboratory examinations revealed the following values: luteinizing hormone (LH) peak, 42.2 $\mathrm{mIU} / \mathrm{mL}$; follicle-stimulating hormone (FSH) peak, 16.8 $\mathrm{mIU} / \mathrm{mL}$; and serum estradiol (E2) level, $47.70 \mathrm{pg} / \mathrm{mL}$. No other laboratory results could be attributed to central precocious puberty.

In general, pelvic ultrasound is not necessarily required for central precocious puberty patients, but follow-up pelvic ultrasound was performed for differential diagnosis due to previously diagnosed left kidney agenesis and increased estradiol levels. As a result, we identified left renal agenesis with left hemivaginal obstruction, separated vaginal canal, and cystic dilatation (Fig. 1). Subsequent magnetic resonance imaging (MRI) revealed a uterus didelphys and obstructive hemivagina with hydrocolpos (Fig. 2). On the basis of the results of the imaging study for further evaluation, we diagnosed her as having HWW syndrome.

She underwent treatment with GnRH analogue with leu-

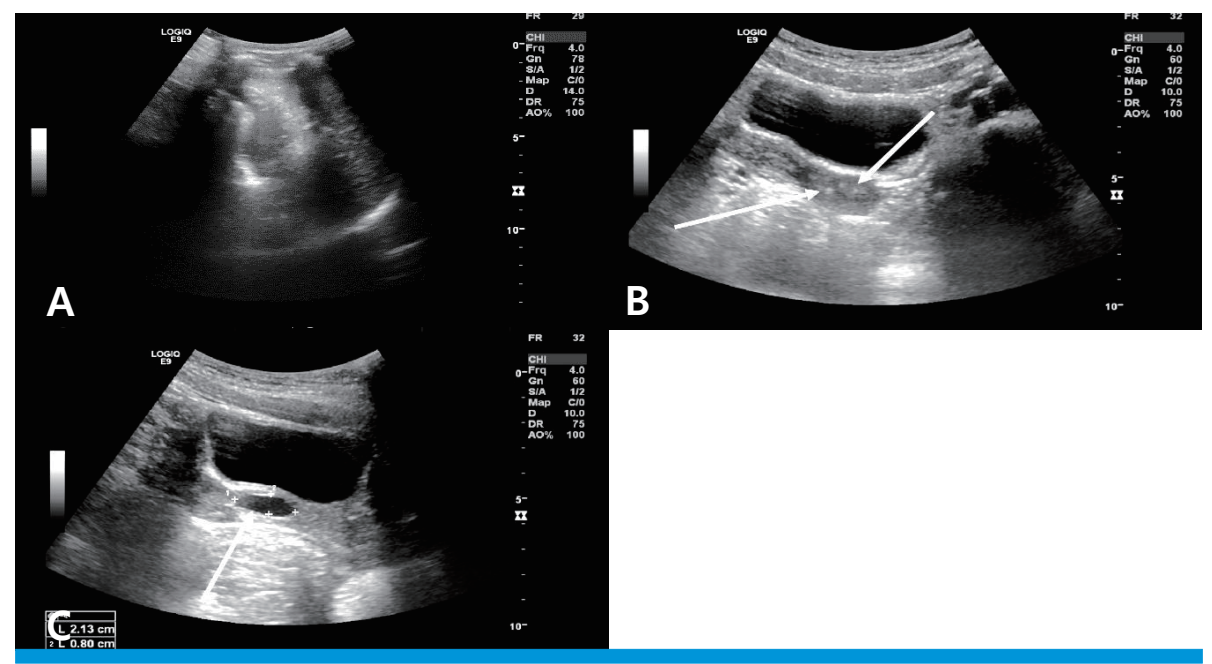

Fig 1. Ultrasonography scan of a 7-year-old girl on August 2017, demonstrating the absence of the left kidney $(A)$ and separated vaginal canal $(B)$. The pelvic ultrasonography scan shows cystic dilatation of the left vagina $(2.13 \times 0.80 \mathrm{~cm})(\mathrm{C})$, and Mullerian duct anomaly is suspected. 
prorelin acetate $90 \mu \mathrm{g} / \mathrm{kg}$ subcutaneously every 28 days from August 2017 to July 2019 at our hospital. In an interim test conducted after 6 months of treatment, her LH level measured 45 min after follow-up GnRH stimulation was $2.3 \mathrm{mIU} / \mathrm{mL}$ (The LH peak level identified in the initial GnRH stimulation test was $42.2 \mathrm{mIU} / \mathrm{mL}$ ). In July 2019, when the $\mathrm{GnRH}$ analogue treatment was terminated, she had an estimated bone age of 12 years, estimated using the Greulich and Pyle method and her chronological age was 10years and 10months. At the end of the GnRH treatment, her height was $152.6 \mathrm{~cm}$ (90th percentile). Her height velocity was maintained at approximately $5 \mathrm{~cm}$ per year during the GnRH analogue treatment. During 24 months of follow-up, no serious problems or complications arose with the patient.

We explained to her parents that HWW syndrome usually presents with dysmenorrhea, pelvic pain, or pelvic mass after menstruation and recommended consultation for surgical treatment before menarche.

\section{Discussion}

This is a case in which HWW syndrome was diagnosed

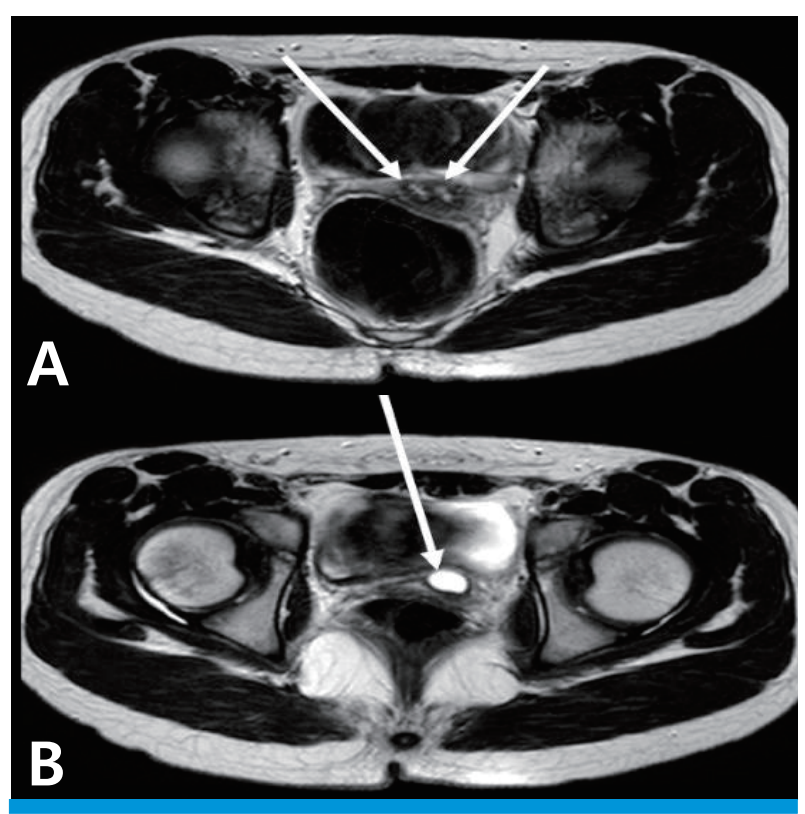

Fig. 2. Magnetic resonance imaging (T2-weighted image) scans suggesting Herlyn-Werner-Wunderlich syndrome. The axial image shows uterus didelphys (arrow) (A). The other axial image shows obstructive hemivagina with hydrocolpos (arrow) (B). through further imaging studies conducted for a patient with central precocious puberty. In general, Mullerian anomalies have not been associated with central precocious puberty. However, if diagnosis is delayed in such cases as this patient, blood reflux into the abdominal cavity may result in endometriosis and may lead to pelvic adhesions and increased risk of abortion or infertility. Therefore, possibilities of genitourinary disease should be checked through history-taking of kidney anomalies and medical examinations. Pelvic ultrasonography can be used to identify changes in the uterus, including autonomic follicular cysts, ovarian or adrenal tumors, and other genitourinary anomalies ${ }^{6)}$. For newborns, ultrasonography plays an important role in identifying Mullerian structures; however, uterine abnormalities are difficult to assess because of the small size and tubular shape of the uterus ${ }^{7)}$. Thus, it is worth considering that patients with an anomaly in the kidney undergo follow up ultrasonography test before the onset of menstruation to detect undiscovered genitourinary diseases such as HWW syndrome.

In our case, the patient had no follow-up visit within 7years after diagnosis of ipsilateral renal agenesis. However, the occurrence of symptoms associated with central precocious puberty led to her visit in our hospital. As a result, an early diagnosis of HWW syndrome was possible through further examinations after checking her past medical history. Through GnRH analogue treatment, we were able to reduce the possibilities of complications associated with HWW syndrome such as dysmenorrhea, endometriosis, pyosalpinx from early menstruation at the same time, along with the treatment of central precocious puberty. If renal anomalies are identified, further screening tests should be conducted prior to menstruation for determining congenital abnormalities of the reproductive tract and vice versa.

\section{Funding}

This research did not receive any specific grant from funding agencies in the public, commercial, or not-forprofit sectors. 


\section{Conflict of interest}

The Author declare that there is no conflict of interest.

\section{ORCID iDs}

Jeeho Han https://orcid.org/0000-0002-5388-0836

Geon Hee Kim https://orcid.org/0000-0003-4955-6512

Su Jin Kim https://orcid.org/0000-0003-0893-0512

\section{Patient consent}

The study was approved by the institutional review board (IRB), and the consent was waived due to the nature of the retrospective study [IRB No. MJH 2019-06-012].

\section{References}

1. Orazi C, Lucchetti MC, Schingo PM, Marchetti P, Ferro F. HerlynWerner-Wunderlich syndrome: uterus didelphys, blind hemivagina and ipsilateral renal agenesis. Sonographic and MR findings in 11 cases. Pediatr Radiol 2007;37:657-65.

2. Gholoum S, Puligandla PS, Hui T, Su W, Quiros E, Laberge JM. Management and outcome of patients with combined vaginal septum, bifid uterus, and ipsilateral renal agenesis (HerlynWerner-Wunderlich syndrome). J Pediatr Surg 2006;41:987-92.

3. Smith NA, Laufer MR. Obstructed hemivagina and ipsilateral renal anomaly (OHVIRA) syndrome: management and followup. Fertil Steril 2007;87:918-22.

4. Wu TH, Wu TT, Ng YY, Ng SC, Su PH, Chen JY, et al. Herlyn-WernerWunderlich syndrome consisting of uterine didelphys, obstructed hemivagina and ipsilateral renal agenesis in a newborn. Pediatr Neonatol 2012;53: 68-71.

5. Zurawin RK, Dietrich JE, Heard MJ, Edwards CL. Didelphic uterus and obstructed hemivagina with renal agenesis: case report and review of the literature. J Pediatr Adol Gynecol 2004;17:37-41.

6. Ziereisen, F., Guissard, G., Damry, N., and Avni, E. F. Sonographic imaging of the paediatric female pelvis. Eur Radiol 2005;15:1296309.

7. Jiwon M. Lee. Herlyn-Werner-Wunderlich Syndrome: A Minireview. Child Kidney Dis 2018;22:12-6. 